\title{
Metabolic Syndrome and Perioperative Complications during Scheduled Surgeries with Spinal Anesthesia
}

\author{
José Pomares' ${ }^{1}$, Gustavo Mora-García ${ }^{2}$, Roberto Palomino', Yéssika De León ${ }^{3}$, \\ Claudio Gómez-Alegría4, Doris Gómez-Camargo 2* \\ ${ }^{1}$ Anesthesiology and Reanimation Department, Faculty of Medicine, University of Cartagena, \\ Cartagena de Indias, Colombia \\ ${ }^{2}$ Tropical Medicine Doctorate Program, Faculty of Medicine, University of Cartagena, \\ Cartagena de Indias, Colombia \\ ${ }^{3}$ Faculty of Medicine, University of Cartagena, Cartagena de Indias, Colombia \\ ${ }^{4}$ Pharmacy Department, Faculty of Sciences, National University of Colombia, Bogotá, Colombia \\ Email: dmtropical@unicartagena.edu.co
}

Received 27 May 2014; revised 29 June 2014; accepted 22 July 2014

Copyright (C) 2014 by authors and Scientific Research Publishing Inc.

This work is licensed under the Creative Commons Attribution International License (CC BY).

http://creativecommons.org/licenses/by/4.0/

C) (i) Open Access

\section{Abstract}

Background: Metabolic syndrome (MS) is a constellation of factors associated with increased risk of developing cardiovascular diseases and Diabetes Mellitus. Despite of the many studies related to MS, little is known about its impact on scenarios such as surgical anesthesia. Objective: To examine the correlation between demographic and metabolic variables with the occurrence of perioperative complications in patients with MS undergoing scheduled surgeries using a spinal anesthesia technique in the surgery department at the University Clinic San Juan de Dios in Cartagena de Indias, Colombia. Methods: Observational, analytical, cross-sectional, single-center study of 150 subjects with MS and 150 control subjects. Perioperative complications, socio-demographic, hemodynamic and respiratory variables were registered. Groups were compared using t test, Fisher's exact test or Chi-square, as appropriate. We applied a logistic multiple regression model, adjusted by backward stepwise at 0.25 and forward at 0.05 , to find possible incompatible associations. $p$ value $<0.05$ was considered significant. Results: There were significant differences between groups in age, American Society of Anesthesiologists physical status classification, frequency of diseases associated to MS and perioperative complications. There were no cases of mortality among patients. There was statistically significant difference between the two groups for intraoperative hypotension and hypertension with $p$ values of $<0.0001$ and 0.034 . Among postoperative complications there was statistically significant difference in pain $(13.3 \%$ vs $5.3 \%$ in patients without MS) and nausea and/or postoperative vomiting ( $8 \%$ vs $2 \%$ in patients without MS) with a 
p value of 0.027 and 0.015 (by Fisher) respectively. Conclusions: Metabolic abnormalities in MS are a risk factor for developing complications in the perioperative period of patients scheduled for surgeries using the subarachnoid anesthesia technique. Accordingly, it is appropriate to implement health intervention strategies by the surgical team, aiming at their prevention and management.

\section{Keywords}

\section{Metabolic Syndrome X, Insulin Resistance, Spinal Anesthesia, Abdominal Obesity, Perioperative Period}

\section{Introduction}

Metabolic syndrome (MS) is defined by a constellation of physiological, biochemical, clinical, and metabolic factors related to the insulin resistance phenomena [1], first described by Himsworth over 60 years ago [2]; causing hyperinsulinemia and hyperglycemia [3], which directly increase the risk of certain conditions, such as kidney stones, atherosclerotic cardiovascular disease (ASCVD), Type 2 Diabetes Mellitus (T2DM), obesity, high blood pressure and mortality; with an association among all of them [4] [5].

Although there are clinical studies of MS in different populations, classification criteria are limited either by their low correlation in their applicability, or because it is limited to the description of the components of the syndrome and do not establish a direct relationship with the pathophysiological mechanism. In 2003, the American Association of Clinical Endocrinology (AACE) unified a number of criteria for the diagnosis of MS, emphasizing the oral glucose tolerance test (OGTT) [6]. These new criteria are added to those established by the World Health Organization (WHO) [7] and ATP III in 2002 [8], where the role of the MS as a risk factor for ASCVD was highlighted; this importance was subsequently confirmed by the AHA and NHLBI [9] and several international organizations [10]. The presence of this syndrome essentially doubles the risk for ASCVD and is a major target for lifestyle intervention. ACC/AHA guidelines seemingly discount the MS because of a lack of clinical trials that specifically target it with drug therapy. The MS nonetheless remains a major cardiovascular risk factor that needs clinical attention [11]. The International Diabetes Federation (IDF) recognized the need for a simple and easily applicable tool for the syndrome diagnosis that could be universally used. In 2006, the IDF consensus group proposed new criteria (essential and additional) for the definition of MS, where ethnic identity and its variations in the identification of obesity are recognized and focused on the prediction of coronary vascular disease and diabetes [12].

The acute disease and the perioperative period are characterized by a state of insulin resistance manifested as hyperglycemia, and leads to several other metabolic and biochemical changes that negatively affect the final organ function [13] [14].

Hyperglycemia in critically ill patients adversely affects perioperative outcomes of these patients [15]. Achieving euglycemia seems beneficial in certain clinical situations [16] [17], but there is disagreement about the ideal target glycemia, duration of therapy, and modality. Pharmacotherapy, exercise and nutrition to improve insulin sensitivity appear promising, but require further evaluation to confirm its effectiveness in reducing perioperative risk [18].

In Medellín, an urban population of Colombia, Davila et al. reported in 2013 a prevalence of $40.7 \%$ for MS [38]. Currently, there is an increasingly high prevalence of patients with MS reported in national and international series where insulin resistance, defined as a state of reduced biological response to physiological concentrations of insulin [3], is a key component of the syndrome, and appears to be the result of excess abdominal adipose tissue; with increased secretion of free fatty acids (FFAs) and pro-inflammatory factors including alpha tumor necrosis factor (TNFa), IL-6 and other adipokines, all resulting in a defect in the transport of glucose to the level of skeletal muscle, and alterations in lipid metabolism [14] [19] [20].

Patients classified with MS have been studied under the baseline risk of cardiovascular events [21] [22] during activities and/or conventional scenarios; but so far there is no conclusive research literature about the behavior of these patients during surgical procedures involving spinal anesthesia; although our group has executed serious studies to elucidate the behavior pattern in terms of perioperative complications when undergoing general 
anesthesia [23]. Therefore the risk of this population is unknown for submission to this anesthetic technique as there is a knowledge gap in this regard.

The aim of this study was to analyze the correlation between socio demographic and metabolic variables with the occurrence of perioperative complications in patients with MS undergoing elective surgery using the technique of spinal anesthesia in a surgery department of the city of Cartagena, Colombia; comparing it with the behavior of a control group of patients without MS. This allowed us to determine the surgical risk in patients with metabolic, neurohumoral and hemodynamic stress [13] [24] that involves anesthesia of reference.

\section{Materials and Methods}

Alongitudinal study was carried out, in the surgery service at the University Clinic San Juan de Dios (CUSJD for its name in spanish) of Cartagena de Indias, Colombia. Patients were followed up during perioperative period and procedure-related complications were registered as final outcome.

Patients undergoing subarachnoid scheduled procedures were enrolled between April 2009 and April 2010.Inclusion criteria were: subjects of both genders, aged 18 to 80 years, with and without a diagnosis of MS according to the criteria of the International Diabetes Federation (IDF 2006) [21]; physical state I, II and III according to the American Society of Anesthesiologists (ASA) classification [25] [26], with intermediate or low surgical risk according to the ACC/AHA guidelines classification for preoperative assessment of the cardiac patient for non-cardiac surgery in September 2007 [27].

Patients were excluded if they met any of the following criteria: physical status IV, V or VI according to the ASA classification, patient refusal of the anesthetic proposal or to participate in the clinical trial, emergency surgery, application of general anesthesia (inhalation, total intravenous, balanced or combined with regional technique), existence of some criterion (active cardiac conditions) to warrant the postponement of surgery, high surgical risk (vascular surgery) [27].

A total of 300 patients of both sexes undergoing surgery using the subarachnoid anesthesia technique were recruited, according to minimum sample size calculation based in global risk for MS [28] [29], and they were distributed in two study cohorts: I. Exposed (150 patients diagnosed with MS according to the guidelines of the International Diabetes Federation [9] [10] [21]; and II. Non-Exposed (150 patients without MS).

All patients were interviewed before anesthesia to determine their ASA physical status score, and their data was included in a single data collection form. They were selected through a random sampling and the information was entered into a database. Qualitative and quantitative variables were tabulated and analyzed using descriptive statistical tools.

The variables studied in the perioperative period were: physical status according to the ASA [25], risk surgical procedure according to the ACC/AHA 2007 [26] [27], perioperative complications both intraoperative and postoperative, hypotension (decrease $\geq 30 \%$ of the mean baseline blood pressure during $\geq 10$ minutes despite administration of conventional vasopressors type ethylephrine in usual doses-10 mg), hypertension (increase $\geq$ $30 \%$ in the mean baseline blood pressure), hypoxemia (decrease $\geq 10 \%$ of the basal peripheral oxyhemoglobin saturation at admission recorded with pulse oximeter), bleeding (loss of $\geq 30 \%$ of blood volume for the type and patient weight), moderate-severe pain (score $>4$ on verbal numerical pain scale pain, while in the post-anesthesia care unit (PACU), after handling it with NSAIDs, opioids and/or surgical wound infiltration with local anesthetic according to institutional pain management protocol for each surgical procedure), post-operative nausea and/ or vomiting (PONV) (prior prophylactic management according to institutional protocol for each surgical procedure and/or patient's own risk) and mortality.

Conventional non-invasive continuous monitoring was performed: (surface electrocardiography, pulse oximetry, noninvasive blood pressure, mean arterial pressure, levels of exhaled carbon dioxide by capnography in nostrils). Later, the surgical anesthetic procedure was performed using the subarachnoid anesthesia technique by the surgical team on duty, without intervening in the quality or type of anesthetic intervention by the attending anesthesiologist.

For both groups, the anesthetic technique and its monitoring was made with the same regular measuring equipment (same for the two groups of patients) and the same anesthetic supplies and human resources. The anesthesiologist criteria were always respected in the decision making about pre, intra and postoperative interventions. Finally, the format of data collection was completed in simultaneous filling out of the anesthetic registration while in the PACU.

For statistical procedures, quantitative variables were described as the mean \pm SD, while categorical variables 
were described through frequency measures. Bothcohorts were compared using null hypothesis tests, for instance t-student was used for means and for frequency measures we used Chi-squareor Fisher's exact test as appropriate. To establish association strength between outcomes (perioperative complications) and MS, two logistic regression models were executed with a stepwise fit determined by p values $<0.05$ to prevent overfitting. Sociodemographic variables and anthropometric parameters were included as possible confusion variables. The first model was used to analyze intraoperative variables and the second model for postoperative variables. In all cases, Odds Ratios were reported, and their validity was determined by the dispersion in the Confidence Interval of $95 \%$ and the statistical significance was established as p values $<0.05$. All procedures were carried out using a data- base processor software type MedCalc Statistical Software 11.4 [30].

The investigation procedure was approved by the medical ethics committee and research department at the University of Cartagena, and the health services provider where research was performed (CUSJD). All patients participating in the study signed the written informed consent for the institutional embodiment of the surgery and the anesthetic technique, in addition to the forms designed for inclusion in the research project. The type of risk posed for patients when subjected to this study, under Resolution 2378 of June 27th 2008 from the Ministry of Social Protection, is "no risk" [31].

\section{Results}

Results of socio-demographic, anthropometric and perioperative variables for patients included in the study are shown in Table 1. Average values for age, weight, height and body mass index (BMI) for the entire population were 49.7 years, $74.4 \mathrm{~kg}, 164 \mathrm{~cm}, 28 \mathrm{~kg} / \mathrm{m}^{2}$, respectively. There was a more even distribution in terms of gender. Referring to the physical condition according to the ASA, there was statistically significant difference with proportions appearance of $1.3(n=2), 90(n=135)$, and $8.7 \%(n=13)$ for physical status I, II and III respectively in patients with MS and $58.7(n=88), 35.3(n=53)$ and $6 \%(n=9)$ in patients without this condition.

With respect to comorbidities, patients in the exposed cohort reported a total of 354 isolated comorbidities and 69 of these events were found in patients from the non-exposed cohort, evidencing a statistically significant difference. $60.7 \%(n=91)$ patients had hypertension, $82.7 \%(n=124)$ hypertriglyceridemia, $40.7 \%(n=61)$ low HDL, 28.7\% ( $=43)$ hyperglycemia, $46.7 \%(n=70)$ obesity and 37.3\% $(n=56)$ overweigh in the exposed cohort. In the non-exposed cohort $5.3 \%(n=8)$ patients had hypertension, $2 \%(n=3)$ hypertriglyceridemia, $1.3 \%$ $(n=2)$ low HDL, $0.7 \%(n=1)$ hyperglycemia, 7.3\% $(n=11)$ obesity and 29.3\% $(n=44)$ overweigh.

Regarding intraoperative complications, 38 events were observed in the exposed cohort and 5 in the non-exposed cohort, thus showing statistically significant difference favoring the non-exposed cohort with less complications occurrence. During the surgical procedures carried out for the exposed cohort, $14.7 \%(\mathrm{n}=22)$ patients developed hypotension, $0.7 \%(n=1)$ hypoxemia, 6.7\% $(n=10)$ hypertension and 3.3\% $(n=5)$ bleeding. In contrast, 1.3\% $(n=2)$ patients suffered from hypotension and hypertension $(\mathrm{p}<0.001$ and $\mathrm{p}=0.017$ respectively), $0.7 \%(\mathrm{n}=1)$ from bleeding $(\mathrm{p}=0.107)$ and nobody had hypoxemia in the non-exposed cohort $(\mathrm{p}=0.5)$; for individual statistically significant differences.

A total of 37 postoperative complications were reported from the exposed cohort and 13 from the non-exposed cohort, which was a significant difference. In the exposed cohort we found that $1.3 \%(n=2)$ of patients developed hypoxemia, $0.7 \%(n=1)$ hypertension, $13.3 \%(n=20)$ suffered from pain, $8 \%(n=12)$ presented nausea and vomiting, and $1.3 \%(\mathrm{n}=2)$ had a different intraoperative complication; in the same cohort hypotension and bleeding were not observed. In the non-exposed cohort $0.7 \%(\mathrm{n}=1)$ developed hypotension and bleeding $(\mathrm{p}=$ $0.5)$, 5.3\% $(\mathrm{n}=8)$ reported pain $(\mathrm{p}=0.013)$ and $2 \%(\mathrm{n}=3)$ nausea and vomiting $(\mathrm{p}=0.0154)$; hypoxemia, hypertension and other complications were not presented $(\mathrm{p}=0.249,0.5$ and 0.249 respectively) (Table 1 ). Among observable outcomes, hipercapnia was not found during the entire follow up period in any of the cohorts.

It is important to appreciate that the same patient may have two or more concurrent comorbidities or complications within each of the study groups.

With regard to the intraoperative complications of the cohorts we found a positive Odds Ratio (OR) between the independent variables hyperglycemia, presence of overweight, hypertriglyceridemia, and presence of hypertension (Table 2). Female gender seems to be a protective association factor of intraoperative complications under the conditions of this study (OR: 0.32, 95\%CI: [0.12 - 0.88], p = 0.028).

In addition, when analyzing the model regarding postoperative complications, this showed association between the male sex and hypertriglyceridemia variables in relation to presenting complications in the postoperative period (Table 3). In this sense, sex had a value for OR: 0.27 (95\%CI: $0.10-0.71, \mathrm{p}=0.008$ ), height OR: 
Table 1. Comparison of socio demographic, anthropometric and perioperative variables between the study groups.

\begin{tabular}{|c|c|c|c|}
\hline & Positive MS & Negative MS & Value-p \\
\hline Age (Mean) & 59.2 & 40.3 & $<0.0001$ (t-test) \\
\hline \multicolumn{4}{|l|}{ Sex \% (n) } \\
\hline Male & $40.6(61)$ & $39.3(59)$ & \\
\hline Female & $59.4(89)$ & $60.7(91)$ & 0.906 (Fisher) \\
\hline Weight (Mean) & 82.3 & 66.5 & $<0.0001$ (t-test) \\
\hline Height (Mean) & 164.5 & 163.5 & 0.605 (t-test) \\
\hline BMI $^{*}$ (Mean) & 32.0 & 24.0 & $<0.0001$ (t-test) \\
\hline \multicolumn{4}{|l|}{ Physical Status ASA* \% (n) } \\
\hline I & $1.3(2)$ & $58.7(88)$ & \\
\hline II & $90.0(135)$ & $35.3(53)$ & \\
\hline III & $8.7(13)$ & $6.0(9)$ & $<0.0001\left(\mathrm{X}^{2}\right)$ \\
\hline \multicolumn{4}{|l|}{ Comorbidities \% (n) } \\
\hline Hypertension & $60.7(91)$ & $5.3(8)$ & \\
\hline Hypertriglyceridemia & $82.7(124)$ & $2.0(3)$ & \\
\hline Low HDL & $40.7(61)$ & $1.3(2)$ & \\
\hline Hyperglycemia & $28.7(43)$ & $0.7(1)$ & \\
\hline Obesity & $46.7(70)$ & $7.3(11)$ & \\
\hline Overweight & $37.3(56)$ & $29.3(44)$ & $<0.0001\left(X^{2}\right)$ \\
\hline \multicolumn{4}{|c|}{ Intraoperative complications \% (n) } \\
\hline Hypotension & $14.7(22)$ & $1.3(2)$ & 2.279e-5 (Fisher) \\
\hline Hypoxemia & $0.7(1)$ & 0.0 & 0.5 \\
\hline Hypertension & $6.7(10)$ & $1.3(2)$ & 0.017 (Fisher) \\
\hline Bleeding & $3.3(5)$ & $0.7(1)$ & 0.107 (Fisher) \\
\hline \multicolumn{4}{|c|}{ Postoperative Complications \% (n) } \\
\hline Hypotension & 0.0 & $0.7(1)$ & 0.5 \\
\hline Hypoxemia & $1.3(2)$ & 0.0 & 0.249 \\
\hline Hypertension & $0.7(1)$ & 0.0 & 0.5 \\
\hline Bleeding & 0.0 & $0.7(1)$ & 0.5 \\
\hline Pain & $13.3(20)$ & $5.3(8)$ & 0.013 \\
\hline Nausea and Vomiting & $8.0(12)$ & $2.0(3)$ & 0.0154 \\
\hline Other & $1.3(2)$ & 0.0 & 0.249 \\
\hline
\end{tabular}

*BMI: body mass index. ASA: American Society of Anesthesiologists. NS: not significant. CHI: Chi square.

1.08 (95\%CI: 1.01 - 1.15, p = 0.014) and hypertriglyceridemia OR: 2.32 (95\%CI: 1.04 - 5.18, p = 0.040).

There were no cases of death in the study groups of patients.

\section{Discussion}

The risk of developing cardiovascular medium and long-term complications in patients with MS has been largely 
Table 2. Multivariated logistic regression model. Number of observations: 300. OR: Odds ratio. ASA: physical status according to the American Society of Anesthesiologists. MS: Metabolic syndrome, HDL: High density lipoprotein.

\begin{tabular}{cccc}
\hline Intraoperative Complications & OR & $\mathrm{p}>\mathrm{z}$ & [95\% Confidence Interval] \\
\hline Hyperglycemia & 5.13 & 0.0001 & $2.07-12.68$ \\
* Sex & 0.33 & 0.028 & $0.12-0.88$ \\
Weight & 0.97 & 0.142 & $0.94-1.01$ \\
Height & 1.06 & 0.078 & $0.99-1.13$ \\
Overweight & 2.37 & 0.036 & $1.06-5.31$ \\
Physical Status ASA III & 3.07 & 0.065 & $0.93-10.14$ \\
Hypertriglyceridemia & 4.43 & 0.002 & $1.75-11.20$ \\
Low levels of HDL & 0.24 & 0.038 & $0.06-0.93$ \\
High Blood Pressure & 3.99 & 0.001 & $1.70-9.37$ \\
\hline
\end{tabular}

${ }^{*}$ Value assigned to men was 1 and to women was 0 . Ergo, being female ( 0.3 closer to 0$)$ was presented as a protective factor.

Table 3. Multivariated logistic regression model. OR: Odds ratio.

\begin{tabular}{cccc}
\hline $\begin{array}{c}\text { Postoperative } \\
\text { Complications }\end{array}$ & OR & $\mathrm{p}>\mathrm{z}$ & [95\% Confidence Interval] \\
\hline Presence of Overweight & 1.67 & 0.193 & $0.77-3.64$ \\
${ }^{*}$ Sex & 0.27 & 0.008 & $0.10-0.71$ \\
Hypertension & 1.92 & 0.102 & $0.88-4.21$ \\
Height & 1.08 & 0.014 & $1.01-1.15$ \\
Hypertriglyceridemia & 2.32 & 0.040 & $1.04-5.18$ \\
\hline
\end{tabular}

${ }^{*}$ Value assigned to men was 1 and to women was 0 . Ergo, being female ( 0.3 closer to 0$)$ was presented as a protective factor.

studied during perioperative period along with some strategies to reduce such risks [32] [33], leading to the knowledge of MS implications in scenarios such as an intensive care unit and general anesthesia [34], Perioperative metabolic changes have been reviewed in patients undergoing cardiac surgery, in which higher risk of developing complications has been found [35]. In this study we found a relationship between MS and developing perioperative complications, where the presence of hypertriglyceridemia, hypertension and overweight demonstrated the highest association to perioperative complications as risks factors. However, this is an epidemiological study and for that reason, our findings are not conclusive with respect to causality events. Second, age and sex distribution among groups show this is not necessarily a representative sample of patients with and without MS undergoing surgery with spinal anesthesia technique; this could be due to the convenience sampling performed in this study in only one clinic in Cartagena de Indias and not in the totality of health providers where subjects could vary [36].

Glance et al. found a 2-fold increased risk of death for patients undergoing non-cardiac surgery with the modified metabolic syndrome who were super obese $\left(>50 \mathrm{~kg} / \mathrm{m}^{2}\right)$, 2- to 2.5 -fold higher risk of cardiac adverse events and 3- to 7-fold higher risk of acute kidney injury in obese (30 to $39.9 \mathrm{~kg} / \mathrm{m}^{2}$ ), morbidly obese (40 to $49.9 \mathrm{~kg} / \mathrm{m}^{2}$ ) and super obese patients compared to normal-weight (18.5 to $\left.24.9 \mathrm{~kg} / \mathrm{m}^{2}\right)$ patients [37]. In our study, we found patients from the exposed cohort to developed more over all complications in the perioperative period, which also had higher weight and BMI. Other authors have also found the presence of MS to be a significant predictor of perioperative complications after radical nephrectomy (adjusted OR, 1.489; 95\%CI, 1.146 - 1.934), in 3337 patients for which complete data to analyze were available [38]. Mullen et al. found that obesity alone may not always increase perioperative morbidity and mortality [39].

After doing a review, Neligan (2010) stated that it was too early to tell whether metabolic disease truly increases 
perioperative risk due to inadequate data [40]. To summarize, in this study it was found that the metabolic alterations of MS (hyperglycemia, overweight, hypertriglyceridemia and hypertension) are associated with a higher number of intraoperative complications such as hypotension and hypertension in the anesthetic context when undergoing elective surgery using the spinal anesthesia technique, which could be a consequence of the concurrent endothelial dysfunction as was said by Ramsay on 2013 [41]. Similarly patients with hypertriglyceridemia and males have higher risk of postoperative complications (pain not controlled with conventional therapy according to protocols and increased nausea or vomiting postoperatively).

In regard to socio-demographic and anthropometric variables, there was a statistically significant difference between the two cohorts for age, weight and BMI, with p values $<0.0001$; where patients in the exposed cohort were older and heavier, as well as more prevalently distributed in a higher physical status category compared to the non-exposed cohort as was expected.BMI was also found to be overall higher in patients with MS than in patients without MS by Cantiello et al. (2014) with a statistically significant difference, as well as wider waist circumference [42]. The above is a global and local concern in terms of $34.6 \%$ of our national adult population being overweighed and $16.5 \%$ being obese, according to the national survey of nutritional status (ENSIN 2010) [43]. We cannot account for possible surgeon bias in referring patients with MS to surgical treatment, which was beyond the scope of this study.

There were more patients from the exposed cohort with all previous comorbidities, indicating the known relationship between these and MS. There was statistically significant difference between the two cohorts for hypotension and hypertension during surgeries, with p values of 2.279e -5 and 0.017 (Fisher) respectively; not so for bleeding ( $p=0.107$ by Fisher) and hypoxemia $(p=0.5)$, because of the extreme low frequency of these events in the observations. Complications evaluated during the follow up, show no significant difference among the cohorts, but pain, nausea and vomiting were more prevalent in the exposed cohort. Increase of MS prevalence has also been described as a postoperative complication for patients undergoing liver transplantation; patients in the exposed cohort showed higher increase in the intake of total energy and saturated fats and a higher prevalence of complications, especially cardiovascular events, than subjects without metabolic syndrome [44]. Note that in our study the MS suffering or non-MS suffering, reflected in the two study cohorts, were taken as one more independent variable, in order to analyze the existence of association and statistical significance between this and perioperative complications.

Low levels of HDL (less than $40 \mathrm{mg} / \mathrm{dl}$ for both sexes) behaves as a probable protector for intraoperative complications, but its wide confidence interval approximated to neutrality (95\%CI: $0.06-0.93)$, indicates the necessity of a larger sample of subjects to emit concept. Cantiello et al. also found a statistically significant difference in respect to HDL, finding lower levels in patients in the exposed cohort [42]; they concluded that MS patients showed a higher rate of high-grade complications compared with patients without MS ( $p<0.001)$. Female gender is a protective association factor for intraoperative complications. The presentation at the model of physical status ASA III as a risk factor but without $\mathrm{p}$ and CI values indicating significance in the regression model observations (CI: 0.93 - 10.14), leads to thought of a confusion to elucidate in subsequent studies with an increased cohort size. Therefore, these results encourage us to further deepen the study of the effects of MS in the anesthetic-surgical setting. Another limitation is presented by labeling individuals as having or not having an attribute which implies an inevitable loss of information and power, dichotomizing is effectively equivalent to losing a third of the data, but is widely used in clinical practice. This variables dichotomization will also reduce the correlation with the true values and carries uncertainty in defining the cutpoint [45].

Prabhakar et al. examined the operative mortality, stroke, renal failure, deep sternal infection, prolonged ventilation, reoperation and postoperative hospital length of stay for patients with moderate and extreme obesity undergoing coronary artery bypass grafting, finding that extreme obesity significantly increased mortality and morbidity after coronary artery operations by more than $50 \%$ [46]. Although most of our variables did not coincide, in our groups there was no mortality.

Obesity, metabolic syndrome, and the surgical patient was studied by Levin et al. in 2009, suggesting further investigation to better understand the etiology, extent, and perioperative consequences of these conditions, especially as they interact with the metabolic and cardiovascular responses to the stresses of surgery [47]. We agree that further research should be undertaken with regard to health consequences of patients with MS to learn more about the scenarios where it interacts, as it is a determining factor in the occurrence of perioperative complications that increase morbidity and mortality of patients, with all socioeconomic consequences this entails. This lays the foundation for future mediate policies, health interventions and health technologies needed in the short, 
medium and long term that enable us to combat this scourge, the phenomenon known as MS.

\section{Conclusion}

It was found that metabolic alterations of MS (hyperglycemia, overweight, hypertriglyceridemia and hypertension) were associated with a higher number of intraoperative complications (hypotension and hypertension); as patients with hypertriglyceridemia and males had higher risk of postoperative complications (pain and increased nausea or vomiting) in the anesthetic context when undergoing elective surgery using the spinal anesthesia technique.

\section{Acknowledgements}

The authors would like to thank the following people and institutions that have made possible the fulfillment of this project: The vicerectoría de Investigación from the Universidad de Cartagena, to the University Clinic San Juan de Dios, the Universidad Nacional de Colombia-Direcciónde Investigaciónsede Bogotá, and COLCIENCIAS-InstitutoColombiano para el Desarrollo de la Ciencia y Tecnología, for its valuable encouragement to José Pomares, through the scholarship program "Francisco José de Caldas” to support young researchers. The authors also acknowledge the contributions in methodological desing of Enrique Ramos MD, MPH from Universidad de Cartagena.

\section{References}

[1] Kaur, J. (2014) A Comprehensive Review on Metabolic Syndrome. Cardiology Research and Practice, 2014, Article ID: 943162. http://dx.doi.org/10.1155/2014/943162

[2] Himsworth, H.P. (2013) Diabetes Mellitus: Its Differentiation into Insulin-Sensitive and Insulin-Insensitive Types. International Journal of Epidemiology, 42, 1594-1598. http://dx.doi.org/10.1093/ije/dyt203

[3] Mlinar, B., Marc, J., Janez, A. and Pfeifer, M. (2007) Molecular Mechanisms of Insulin Resistance and Associated Diseases. Clinica Chimica Acta, 375, 20-35. http://dx.doi.org/10.1016/j.cca.2006.07.005

[4] Wilson, P.W., D’Agostino, R.B., Parise, H., Sullivan, L. and Meigs, J.B. (2005) Metabolic Syndrome as a Precursor of Cardiovascular Disease and Type 2 Diabetes Mellitus. Circulation, 112, 3066-3072. http://dx.doi.org/10.1161/CIRCULATIONAHA.105.539528

[5] Domingos, F. and Serra, A. (2014) Metabolic Syndrome: A Multifaceted Risk Factor for Kidney Stones. Scandinavian Journal of Urology.

[6] Einhorn, D., Reaven, G.M., Cobin, R.H., Ford, E., Ganda, O.P., Handelsman, Y., et al. (2003) American College of Endocrinology Position Statement on the Insulin Resistance Syndrome. Endocrine Practice, 9, 237-252.

[7] Alberti, K.G. and Zimmet, P.Z. (1998) Definition, Diagnosis and Classification of Diabetes Mellitus and Its Complications. Part 1: Diagnosis and Classification of Diabetes Mellitus Provisional Report of a WHO Consultation. Diabetic Medicine, 15, 539-553. http://dx.doi.org/10.1002/(SICI)1096-9136(199807)15:7<539::AID-DIA668>3.0.CO;2-S

[8] Expert Panel on Detection, Evaluation, and Treatment of High Blood Cholesterol in Adults (2001) Executive Summary of the Third Report of the National Cholesterol Education Program (NCEP) Expert Panel on Detection, Evaluation, and Treatment of High Blood Cholesterol in Adults (Adult Treatment Panel III). JAMA, 285, 2486-2497. http://dx.doi.org/10.1001/jama.285.19.2486

[9] Grundy, S.M., Cleeman, J.I., Daniels, S.R., Donato, K.A., Eckel, R.H., Franklin, B.A., et al. (2005) Diagnosis and Management of the Metabolic Syndrome: An American Heart Association/National Heart, Lung, and Blood Institute Scientific Statement. Executive Summary. Cardiology in Review, 13, 322-327.

[10] Alberti, K.G., Eckel, R.H., Grundy, S.M., Zimmet, P.Z., Cleeman, J.I., Donato, K.A., et al. (2009) Harmonizing the Metabolic Syndrome: A Joint Interim Statement of the International Diabetes Federation Task Force on Epidemiology and Prevention; National Heart, Lung, and Blood Institute; American Heart Association; World Heart Federation; International Atherosclerosis Society; and International Association for the Study of Obesity. Circulation, 120, 16401645. http://dx.doi.org/10.1161/CIRCULATIONAHA.109.192644

[11] Stone, N.J., Robinson, J., Lichtenstein, A.H., BaireyMerz, C.N., Lloyd-Jones, D.M., Blum, C.B., et al. (2013) 2013 ACC/AHA Guideline on the Treatment of Blood Cholesterol to Reduce Atherosclerotic Cardiovascular Risk in Adults: A Report of the American College of Cardiology/American Heart Association Task Force on Practice Guidelines. Journal of the American College of Cardiology.

[12] Alberti, K.G., Zimmet, P. and Shaw, J. (2007) International Diabetes Federation: A Consensus on Type 2 Diabetes Prevention. Diabetic Medicine, 24, 451-463. http://dx.doi.org/10.1111/j.1464-5491.2007.02157.x 
[13] Seematter, G., Binnert, C., Martin, J.L. and Tappy, L. (2004) Relationship between Stress, Inflammation and Metabolism. Current Opinion in Clinical Nutrition \& Metabolic Care, 7, 169-173. http://dx.doi.org/10.1097/00075197-200403000-00011

[14] Wells, G.D., Noseworthy, M.D., Hamilton, J., Tarnopolski, M. and Tein, I. (2008) Skeletal Muscle Metabolic Dysfunction in Obesity and Metabolic Syndrome. The Canadian Journal of Neurological Sciences, 35, 31-40.

[15] Donatelli, F., Cavagna, P., Di, D.G., Catenacci, A., Di, N.M., Lorini, L., et al. (2008) Correlation between Pre-Operative Metabolic Syndrome and Persistent Blood Glucose Elevation during Cardiac Surgery in Non-Diabetic Patients. Acta Anaesthesiologica Scandinavica, 52, 1103-1110. http://dx.doi.org/10.1111/j.1399-6576.2008.01693.x

[16] Bagry, H.S., Raghavendran, S. and Carli, F. (2008) Metabolic Syndrome and Insulin Resistance: Perioperative Considerations. Anesthesiology, 108, 506-523. http://dx.doi.org/10.1097/ALN.0b013e3181649314

[17] Goel, N., Gupta, K., Bhadoria, P. and Anand, R. (2010) Anaesthetic Considerations in Metabolic Syndrome X. Indian Journal of Anaesthesia, 54, 74-75. http://dx.doi.org/10.4103/0019-5049.60512

[18] Mechanick, J.I., Kushner, R.F., Sugerman, H.J., Gonzalez-Campoy, J.M., Collazo-Clavell, M.L., Guven, S., et al. (2008) American Association of Clinical Endocrinologists, The Obesity Society, and American Society for Metabolic \& Bariatric Surgery Medical guidelines for Clinical Practice for the Perioperative Nutritional, Metabolic, and Nonsurgical Support of the Bariatric Surgery Patient. Endocrine Practice, 14, 1-83. http://dx.doi.org/10.4158/EP.14.S1.1

[19] Kuchta, K.F. (2005) Pathophysiologic Changes of Obesity. Anesthesiology Clinics of North America, 23, 421-429. http://dx.doi.org/10.1016/j.atc.2005.03.004

[20] López-Jaramillo, P., Pradilla, L.P. and Bracho, Y. (2005) Papel del Adipocito en la Inflamación del Síndrome Metabólico. Acta Medica Colombiana, 30, 137-140.

[21] Alberti, K.G., Zimmet, P. and Shaw, J. (2006) Metabolic Syndrome-A New World-Wide Definition. A Consensus Statement from the International Diabetes Federation. Diabetic Medicine, 23, 469-480. http://dx.doi.org/10.1111/j.1464-5491.2006.01858.x

[22] Whelan, D.M. and Roy, R.C. (2006) Diseases of Aging That Emerge From the Metabolic Syndrome. Anesthesiology Clinics of North America, 24, 599-619. http://dx.doi.org/10.1016/j.atc.2006.05.008

[23] Pomares, J., Palomino, R., Gómez, C.J. and Gómez-Camargo, D. (2012) Síndrome Metabólico y Complicaciones Perioperatorias Durante Cirugías Programadas con Anestesia General. Revista Colombiana de Anestesiología, 40, 106112. http://dx.doi.org/10.1016/S0120-3347(12)70022-9

[24] Seematter, G., Binnert, C. and Tappy, L. (2005) Stress and Metabolism. Metabolic Syndrome and Related Disorders, 3, 8-13. http://dx.doi.org/10.1089/met.2005.3.8

[25] Keats, A.S. (1978) The ASA Classification of Physical Status-A Recapitulation. Anesthesiology, 49, $233-236$. http://dx.doi.org/10.1097/00000542-197810000-00001

[26] Saklad, M. (1941) Grading of Patients for Surgical Procedures. Anesthesiology, 2, 281-284.

[27] Fleisher, L.A., Beckman, J.A., Brown, K.A., Calkins, H., Chaikof, E.L., Fleischmann, K.E., et al. (2007) ACC/AHA 2007 Guidelines on Perioperative Cardiovascular Evaluation and Care for Noncardiac Surgery: A Report of the American College of Cardiology/American Heart Association Task Force on Practice Guidelines (Writing Committee to Revise the 2002 Guidelines on Perioperative Cardiovascular Evaluation for Noncardiac Surgery) Developed in Collaboration with the American Society of Echocardiography, American Society of Nuclear Cardiology, Heart Rhythm Society, Society of Cardiovascular Anesthesiologists, Society for Cardiovascular Angiography and Interventions, Society for Vascular Medicine and Biology, and Society for Vascular Surgery. Journal of the American College of Cardiology, 50, e159-e242. http://dx.doi.org/10.1016/j.jacc.2007.09.003

[28] Cohen, J. (1992) Statistical Power Analysis. Current Directions in Psychological Science, 1, 98-101. http://dx.doi.org/10.1111/1467-8721.ep10768783

[29] Botero, J.C., Salazar, D. and Cortez, O. (2006) Metabolic Syndrome and Cardiovascular Risk. Síndrome Metabólico y Riesgo Cardiovascular. Ces Medicina, 20, 73-81.

[30] MedCalc Statistical Software [Computer Program]. Version 11.5.0, (c)1993-2011. 2011. http://www.medcalc.org/

[31] Ministerio de la Protección Social (2008) Resolución Número 2378 de 2008. Por la cual se adoptan las Buenas Prácticas Clínicas para las instituciones que conducen investigación con medicamentos en seres humanos. Diario oficial de la República de Colombia 47033 on June 27th 2008.

[32] Gage, M.J., Schwarzkopf, R., Abrouk, M. and Slover, J.D. (2014) Impact of Metabolic Syndrome on Perioperative Complication Rates after Total Joint Arthroplasty Surgery. The Journal of Arthroplasty, in press.

[33] Cho, H., Yoshikawa, T., Oba, M., Hirabayashi, N., Shirai, J., Aoyama, T., et al. (2014) Matched Pair Analysis to Examine the Effects of a Planned Preoperative Exercise Program in Early Gastric Cancer Patients with Metabolic Syndrome to Reduce Operative Risk: The Adjuvant Exercise for General Elective Surgery (AEGES) Study Group. Annals of Surgical Oncology, 21, 2044-2050. http://dx.doi.org/10.1245/s10434-013-3394-7 
[34] Campillo Acosta, D., Berdasquera Corcho, D. and Coronado Mestre, R. (2007) Mortalidad Asociada al Síndrome Metabólico. Revista Cubana de Medicina General Integral, 23. http://scielo.sld.cu/scielo.php?script=sci_arttext\&pid=S0864-21252007000200003\&lng=es\&nrm=iso

[35] Jakob, S.M. and Stanga, Z. (2010) Perioperative Metabolic Changes in Patients Undergoing Cardiac Surgery. Nutrition, 26, 349-353. http://dx.doi.org/10.1016/j.nut.2009.07.014

[36] Hedt, B.L. and Pagano, M. (2011) Health Indicators: Eliminating Bias from Convenience Sampling Estimators. Statistics in Medicine, 30, 560-568.

[37] Glance, L.G., Wissler, R., Mukamel, D.B., Li, Y., Diachun, C.A., Salloum, R., Fleming, F.J. and Dick, A.W. (2010) Perioperative Outcomes among Patients with the Modified Metabolic Syndrome Who Are Undergoing Noncardiac Surgery. Anesthesiology, 113, 859-872. http://dx.doi.org/10.1097/ALN.0b013e3181eff32e

[38] Selph, J.P., Whited, W.M., Smith, A.B., Matthews, J., Pruthi, R.S., Wallen, E.M., Nielsenx, M.E. and Woods, M.E. (2014) Metabolic Syndrome as a Predictor for Postoperative Complications after Urologic Surgery. Urology, 83, 1051-1059. http://dx.doi.org/10.1016/j.urology.2014.01.014

[39] Mullen, J.T., Moorman, D.W. and Davenport, D.L. (2009) The Obesity Paradox: Body Mass Index and Outcomes in Patients Undergoing Nonbariatric General Surgery. Annals of Surgery, 250, 166-172. http://dx.doi.org/10.1097/SLA.0b013e3181ad8935

[40] Neligan, P.J. (2010) Metabolic Syndrome: Anesthesia for Morbid Obesity. Current Opinion in Anaesthesiology, 23, 375-383. http://dx.doi.org/10.1097/ACO.0b013e328338caa0

[41] Ramsay, M.A. (2013) The Chronic Inflammation of Obesity and Its Effects on Surgery and Anesthesia. International Anesthesiology Clinics, 51, 1-12. http://dx.doi.org/10.1097/AIA.0b013e3182981219

[42] Cantiello, F., Cicione, A., Autorino, R., De Nunzio, C., Salonia, A., Briganti, A., Aliberti, A., Perdonà, S., Tubaro, A. and Damiano, R. (2014) Metabolic Syndrome, Obesity, and Radical Cystectomy Complications: A Clavien Classification System-Based Analysis. Clinical Genitourinary Cancer, in press.

[43] lvarez, M., Estrada, A., Fonseca, Z., Forero, Y., Gempeler, J., Heredia, A., et al. (2011) Encuesta nacional de la situación nutricional en Colombia 2010-ENSIN-/ICBF-Instituto Colombiano de Bienestaar Familiar. ICBF-Instituto Colombiano de Bienestar Familiar, Bogotá.

[44] Lunati, M.E., Grancini, V., Agnelli, F., Gatti, S., Masserini, B., Zimbalatti, D., Pugliese, G., Rossi, G., Donato, M.F., Colombo, M., Beck-Peccoz, P. and Orsi, E. (2013) Metabolic Syndrome after Liver Transplantation: Short-Term Prevalence and Pre- and Post-Operative Risk Factors. Digestive and Liver Disease, 45, 833-839. http://dx.doi.org/10.1016/j.dld.2013.03.009

[45] Royston, P., Altman, D.G. and Sauerbrei, W. (2006) Dichotomizing Continuous Predictors in Multiple Regression: A Bad Idea. Statistics in Medicine, 25, 127-141. http://dx.doi.org/10.1002/sim.2331

[46] Prabhakar, G., Haan, C.K., Peterson, E.D., Coombs, L.P., Cruzzavala, J.L. and Murray, G.F. (2002) The Risks of Moderate and Extreme Obesity for Coronary Artery Bypass Grafting Outcomes: A Study from the Society of Thoracic Surgeons’ Database. Annals of Thoracic Surgery, 74, 1125-1130. http://dx.doi.org/10.1016/S0003-4975(02)03899-7

[47] Levin, P.D. and Weissman, C. (2009) Obesity, Metabolic Syndrome, and the Surgical Patient. Anesthesiology Clinics, 27, 705-719. http://dx.doi.org/10.1016/j.anclin.2009.09.006 
Scientific Research Publishing (SCIRP) is one of the largest Open Access journal publishers. It is currently publishing more than 200 open access, online, peer-reviewed journals covering a wide range of academic disciplines. SCIRP serves the worldwide academic communities and contributes to the progress and application of science with its publication.

Other selected journals from SCIRP are listed as below. Submit your manuscript to us via either submit@scirp.org or Online Submission Portal.
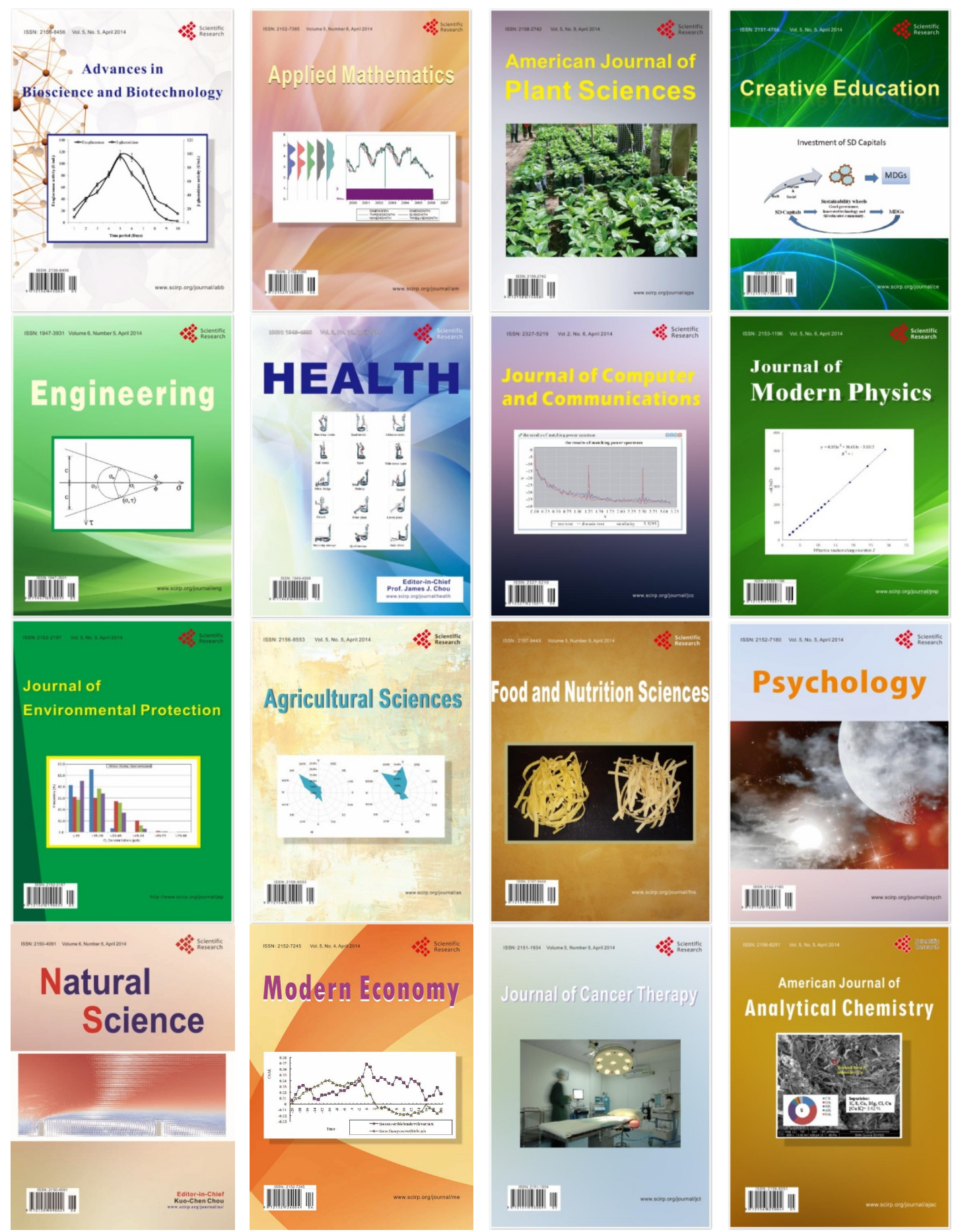